\title{
Mobility of Interphase Boundaries and Hysteresis of Martensitic Transformation in Alloys
}

\author{
Yu.N. Koval \\ Institute of Metal Physics, Phase Transformation Department, Nat. Acad. Sc. Ukr., Vernadsky St. 36, \\ 252142.Kiev, Ukraine
}

\begin{abstract}
A phenomenological description of martensitic transformation is proposed on the basis of such parameters as: a degree of lattice coherency between austenite and martensite phases, mobility of interphase boundaries, elastic energy stored during martensitic transformation and degree of its relaxation. Som? experimental results confirming the proposed ideas are presented.
\end{abstract}

\section{INTRODUCTION}

Assuming that thermoelastc balance in fulfilled, a martensitic transition can be characterized by the following features [1]:

- high mobility of interphase boundaries provided by the coherency between parent and martensite phases;

- availability of transformation stresses in the austenite-martensite system;

- two-phase equilibrium state

As to the last feature, according to Gibbs phase rule, such a type of equilibrium is impossible in a onccomponent system [2]. Hence, to satisfy the requirements of thermoelastic balance it is necessary to take into account some inhomogeneities (concentration inhomogeneities, local fluctuations of long-range order, etc.), which are really present in the high temperature parent phase. However, the more important condition for realization of thermoelastic equilibrium is the availability of long-range elastic stresses in ensemble of martensitic crystals and static internal stresses caused by dislocation arrays and other sources.

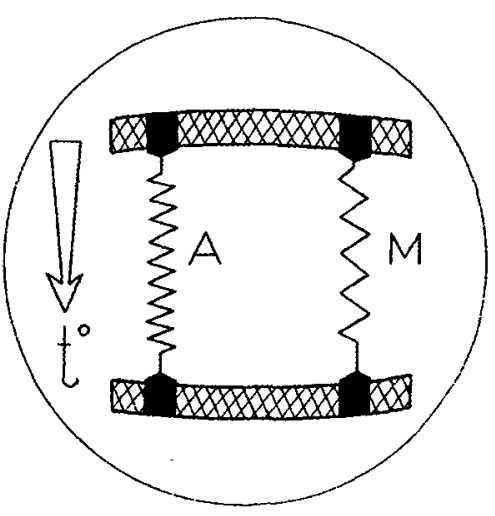

Figure 1: Model of martensitic transformation in a medium with heterogeneities.

Rheologic model for the martensitic transformation (MT) with possibility of two-phase coexistence is presented in Fig. 1, where one region contains martensite phase, and the other (high temperature phase). As shown, interaction between phases is provided in a flexible elastic way.

Transformation stresses will be accommodated during MT in a self-consistent way. In the zeroth order approximatio., hysteresis can be neglected in the systems with a pronounced thermoelastic nature of MT and narrow range of temperature hysteresis. Under these conditions the problem can be reduced to investigation of two-phase equilibrium by taking into account availability of heterogeneous internal stresses. As it has been shown in [3], the thermoelastic equilibrium state can be described by means of a non-linear self-consistent equation for the internal stresses. Solutions of the equation obtained by the method proposed allow to describe microscopic and macroscopic characteristics of heterogeneous structure as a function of temperature under conditions of thermoelastic equilibrium. 


\section{MOBUITY OF INTERFACE BOUNDARIES AND HYSTERESIS OF MARTENSITIC TRANSFORMATION}

Alccording to the principle of cooperative ordering, phase coherency is the other main characteristic in theory of MT. It cannot be directly obtained from experimental data by means of crystallographic investigations. Nevertheless, assumption about availability of coherency and coherent interaction among phases can be imposed on the basis of a high growth rate of martensite crystals. Moreover, it can be supported by observation of thermoelastic nature of MT. In practice, half-coherent boundaries are mostly observed, where coherent regions are interlaced by incoherent ones. In incoherent regions gradually accumulated incongruity relaxes owing to creation of discontinuity (dislocations).

Because MT mainly takes place by means of reversible movement of interface boundaries, it can be characterized by the following parameters:

- lattice soherency between high and low temperature phases;

- mobility of interface boundaries;

- elastic stresses and especially their distribution;

- character of relaxation processes.

The coherency coefficient, measuring the degree of coherency between lattices of parent and martensitic phases, is related to lattice spacings of both phases and orientation of a habit plain between the phases. There are experimental data on direct resolution of crystal lattices for thermoelastic martensite showing appearance of misfit dislocations [4]. According to [5], the value of additional shear with invariant lattice during MT is a convenient and exhaustive characteristic feature for estimation of lattice coherence between both phases.

Another method of estimation of lattice coherency between parent and martensite phases could be proposed. MT is known [6] to be characterized by distortion $\hat{\varepsilon}$ and strain $\hat{e}$, symmetric part of distortion for small strains:

$$
e_{i j}=\frac{1}{2}\left(\varepsilon_{i j}+\varepsilon_{j i}\right) \text {. }
$$

Let parent and martensitic phases be divided by a plane boundary with normal vector $\vec{n}$. A tensor cf "effective" martensitic strain $\hat{d}$ can be defined by the following relationship:

$$
d_{i j}=n_{i} n_{k} e_{k j}+n_{j} n_{k} e_{k i}-n_{i} n_{j} e_{k k} \text {, }
$$

where summation over repetitive indices is assumed. It should be noted from the expression (2), that $\hat{d}$ is a strain with invariant plane $\vec{n}$. Moreover, if a plane $\vec{n}$ is an invariant one for a strain $\hat{e}$, i.e., if

$$
\varepsilon_{i j}=n_{i} \varepsilon_{j},
$$

then tensors $\hat{e}$ and $\hat{d}$ are equal.

In the approximation of homogeneous isotropic medium a force applied to coherent interface boundary and caused by its own elastic stresses is dependent on combination of scalar invariants of tensors $\hat{e}$ and $\hat{d}$. If tensors $\hat{e}$ and $\hat{d}$ are equal, then static elastic stresses caused by existence of interface boundary vanish, as it does during deformation with invariant plane. However, the more difference between a transformation strain and a strain with an invariant plane $\vec{n}$, the stronger static elastic stress is caused by coherent boundary.

A transformation strain can be brought to a strain with invariant plane by means of an additional strain with invariant lattice. Thus, elastic stresses on an interface boundary can be partially or fully removed, but it leads to appearance of dislocations or twins on the interface boundary. In any case, a value measuring difference between tensors $\hat{e}$ and $\hat{d}$ could be used for estimation of misfitting between parent and martensitic phases.

Two mutually independent scalar variables of degree 2 can be composed on the basis of components of a symmetric tensor. For example, they can be implemented by either summation of the squares of diagonal components:

$$
e_{1}=e_{i i}^{2}, \quad d_{1}=d_{i i}^{2},
$$


or by summation of the squares of all tensor components except for the diagonal components (4):

$$
e_{2}=e_{i j} e_{i j}-e_{i i}^{2}, \quad d_{2}=d_{i j} d_{i j}-d_{i i}^{2} .
$$

Then the misfitting coefficients can be expressed as follows:

$$
k_{1}=e_{1}-d_{1}, \quad k_{2}=e_{2}-d_{2},
$$

where $k_{1}$ is accounts for dilatation effects and $k_{2}$ is related to the shear component of the transformation stress.

Thermoelastic equilibrium between phases can be described according to [7] as:

$$
\Delta G_{\text {chem }}+\eta \Delta G_{e l}= \pm v_{m} \tau_{0} \gamma_{T},
$$

where $\Delta G_{c h e m}$ is the molar free energy difference, $\Delta G_{e l}$ the elastic energy associated to the creation and growth of martensite in the parent phase, $v_{m}$ the molar volume of material, $\tau_{0}$ the shear stress needed for moving an interphase boundary, $\gamma_{T}$ the shear strain during MT and $\eta$ a factor which is dependent on geometry of a martensite crystal. The parameter $\tau_{0}$ is assumed to be a function of both mobility of an interphase boundary $c_{G}$ (or lattice coherency $c_{D}$, because in the first approximation $c_{G}=c_{D}^{-1}$ ) and of elastic stresses caused by austenite-martensite interaction:

$$
\tau_{0}=f\left(c_{D}, E_{e l}\right)
$$

An explicit representation of the relationship (8) is known to be a very hard problem, but from the comparison of experimental values of $\tau_{0}$ for various types of MT it can be obtained that:

- the more lattice misfitting between parent and martensitic phases, the more undercooling below $T_{0}$ is necessary for appearance of initial martensite crystals, i.e. the value $\Delta T=T_{0}-M_{\mathcal{S}}$ is less for thermoelastic martensite than for MT with wide temperature hysteresis;

In the first approximation, the degree of undercooling, which is necessary for the beginning of direct (reverse) $M T$, is assumed to be proportional to $c_{D}$. Then,

$$
\Delta G_{M T}=\alpha c_{D}
$$

where $\alpha$ is a coefficient of proportionality which reconcile of left and right parts of the equation with different dimensions; $\Delta G_{M T}$ is thermodynamic potential difference corresponding to the beginning of the direct (reverse) MT. The following expressions can be respectively written for the direct and the reverse MT:

$$
\Delta G_{M T}^{d}=\Delta S_{M T} \Delta T_{D T}=\Delta S_{M T}\left(T_{0}-M_{S}\right), \quad \Delta G_{M T}^{r}=\Delta S_{M T} \Delta T_{R T}=\Delta S_{M T}\left(A_{S}-T_{0}\right) .
$$

Since $\left(\Delta G_{M T}^{d}=\Delta G_{M T}^{r}\right)$, of equations in (10) given:

$$
2 \Delta G_{M T}=\Delta S_{M T}\left(A_{s}-M_{S}\right) .
$$

It can be assumed that $\Delta T_{M T} \sim\left(A_{s}-M_{S}\right)$, and then:

$$
\Delta T_{M T}=2 \alpha \frac{c_{D}}{\Delta S_{M T}} .
$$

It is necessary to determine the physical meaning of the coefficient $\alpha$. Martensite crystal growth begins from the creation of a martensite with the critical nucleus size and it continues in the whole martensite temperature range (really, it is true for direct MT at least). Then, the coefficient $\alpha$ can be considered as equal to the nucleation energy of martensite with the critical nucleus size:

$$
\Delta T_{M T}=2 \varepsilon \frac{c_{D}}{\Delta S_{M T}}=2 \varepsilon \frac{c_{D} T_{0}}{\Delta H_{M T}},
$$

where $T_{0}$ is the equilibrium temperature between parent and martensitic phases, $\Delta H_{M T}$ is the enthalpy of MT.

Thus, the value of hysteresis is directly proportional to both the coefficient $c_{D}$ and the nucleation energy of martensite with critical nucleus, and it is inversely proportional to the entropy difference during 
MT. Therefore, the value of temperature hysteresis can be changed by means of changing $c_{D}$, because $\varepsilon$ and $\Delta S_{M T}$ are assumed to be changed insignificantly. It can be proved by the following experimental results.

In Fig. 2 the relationships of characteristic temperatures $\left(M_{s}, M_{f}, A_{s}, A_{f}\right)$ and flexures $(\delta)$ for triplepoint loading are shown as a function of temperature and number of MT cycles (for the 1st cycle and the Sth one) for the alloy Fe- $\mathrm{Ni} \mathrm{31 \% -Nb} \mathrm{3 \%} \mathrm{[7].} \mathrm{As} \mathrm{it} \mathrm{is} \mathrm{seen} \mathrm{in} \mathrm{the} \mathrm{figure,} \mathrm{significant} \mathrm{decrease} \mathrm{of} \mathrm{temperature}$ $A_{s}\left(\sim 150^{\circ} \mathrm{C}\right)$ and full recovery of shape takes place.

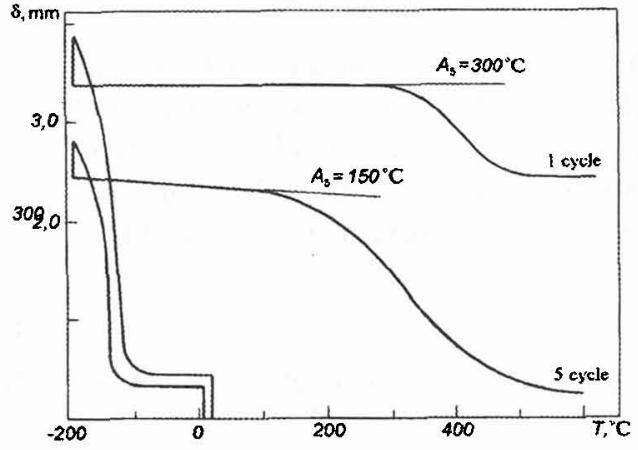

Figure 2: Characteristic temperature changes $\left(M_{s}, M_{f}, A_{s}, A_{f}\right)$ and flexures $(\delta)$ for triple-point loading as functions of temperature and number of MT cycles for the alloy Fe$\mathrm{Ni} 31 \%-\mathrm{Nb} 3 \%$.

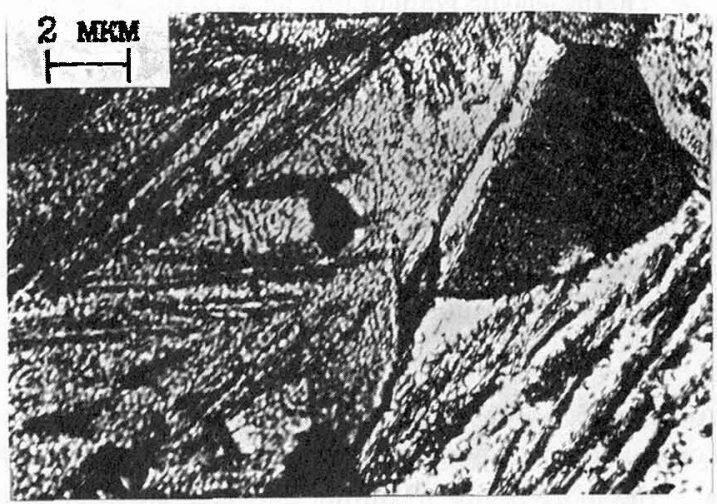

Figure 3: Structure of austenite after one cycle of $\gamma \leftrightarrow \alpha$ transformation. The upper layer of width $0.2-0.3 \mathrm{~mm}$ has been removed.

It is essential feature for this alloy, that additional shear during deformation with invariant lattice takes place by gliding complete dislocations with a Burgers vector $1 / 2(111)$ in planes $\{112\}$. During the reverse transformation dislocations glide in planes $\{311\}$ (8 variants) and $\{202\}$ (4 variants). Althougli dislocations are complete and typical $(1 / 2<110>)$, they are situated in the other planes that are commoriy specific for them. Therefore, these dislocations are characterized by a high energy and, that is why, they tend to decrease energy by means of mutual interaction. As a result, dislocations can be pinned. In fact, appearance of oriented dislocation facets leads to putting out deformation with invariant lattice. Finally, significant decrease of hysteresis and increase of interface boundary mobility are observed with it consequent shape memory effect. This statement is supported by existence of needles in austenite after one full cycle of MT (Fig. 3).

\section{INFLUENCE OF ELASTIC STRESSES STORED DURING DIRECT MT ON REVERSE MT}

Direct influence of elastic stresses (related to creation of martensite crystals) on reverse MT is manifesteil by a full shape memory effect for the binary alloy $\mathrm{Fe}-32.8 \% \mathrm{wt}$. $\mathrm{Ni}$, although this alloy usually demonstrates $40 \%$ shape recovery effect. The preliminary condition in the heat treatment of the alloy, which excludes any direct influence of additional stresses of thermal nature. Next the alloy was subjected to a temperature cycling heatment in the range $\left(M_{s} \div<A_{s}\right)$ As a result, appropriate conditions appear for direct influence of martensitic transformation stresses (caused by new martensite crystals) on driving force of reverse MT (on the one hand) and reverse movement of interface boundary (on the other hand). Finally, it provides narrow hysteresis during MT (in comparison with ordinary value $\Delta T \sim 400^{\circ} \mathrm{C}$ for such an alloy) and full shape recovery (Fig. 4).

The other way of ruling by MT characteristics consists in repression of relaxation stresses stored during direct martensitic transformation. Some types of relaxation should be distinguished:

- dynamic relaxation during direct MT;

- static relaxation, i.e. thermally controlled decrease of stresses after the MT is finished. 
Dynamic relaxation is mainly dependent on the type of martensitic transformation, and static relaxation on strength properties of material, temperature, holding time after MT and rate of heating up to temperature of reverse transformation.

In Fig. 5 the full record of temperature hysteresis during MT is presented according to experimental data obtained by measurements of electric resistance (alloy $\mathrm{Cu}-\mathrm{Sn}$ ). For all of the three cycles the cooling rate was equal to $4 \% \mathrm{~min}$. For the first and third cycles the heating rate was approximately equal to $100 \% \mathrm{~min}$. For the second cycle it was barely equal to $90 \% \mathrm{~min}$ in the static relaxation range (less than $\left.20^{\circ} \mathrm{C}\right)$ and it was slightly increased $\left(\sim 100^{\circ} / \mathrm{min}\right)$ in the temperature range where precipitation processes can be possible. In the first and third cycles the maximal repression of the static stress relaxation was reached. Nevertheless, relaxation took place in the second cycle, but according to observed values of $M_{s}$ and $M_{f}$ relaxation was not followed by irreversible change of a "chemical" constituent because of precipitation. As it has been shown in Fig. 5, in the second cycle temperatures $A_{s}$ and $A_{f}$ increased up to higher values $-10^{\circ} \mathrm{C}$ and $32^{\circ} \mathrm{C}$ in comparison with the first and third cycles, where they were equal $-125^{\circ} \mathrm{C} \pm 5^{\circ} \mathrm{C}$ and -$15^{\circ} \mathrm{C} \pm 5^{\circ} \mathrm{C}$ respectively.

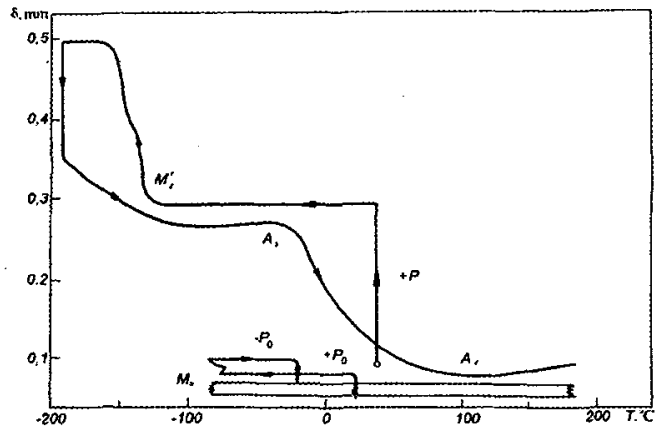

Figure 4: Flexure $(\delta)$ as a function of temperature for the binary alloy Fe-32.8\% wt. Ni. Preliminary heat treatment is partial $\gamma \rightarrow \alpha$ transformation with applied load $P_{0}, 10$ cycles of $\gamma \leftrightarrow \alpha$ transformations without loading.

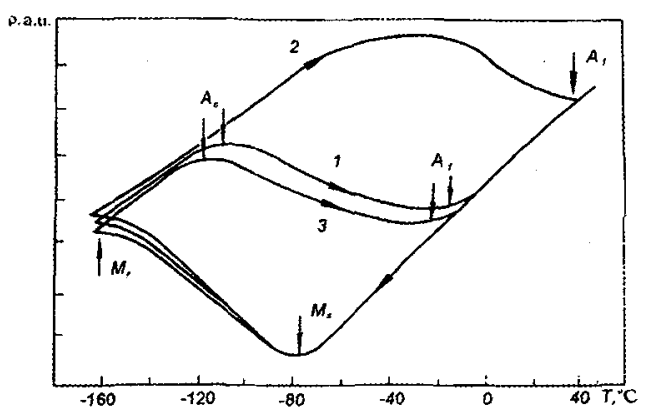

Figure 5: Electric resistance $(\rho)$ for consequent cycles (1-3) of martensitic transformation.

In all the aforementioned experiments the stored elastic energy was generally manipulated by variation of heating rate. The question naturally rises about other ways of influence on parent material for increasing mobility of interface boundaries under ordinary heating rates. For this purpose, additional sources of internal stresses, which are similar to the stresses stored during MT, would be created. Numerous experimental results have shown that precipitation particles can do as the stress sources, provided they are coherent with austenite and coherency is stable during the following MT. The latter means that the precipitates are coherent with martensite crystals too.

Particles, as memory units, are homogeneously distributed in martensite, and the elastic energy stored by the particles can be described by the following expression:

$$
E=c \mu \varepsilon^{2}(a / b)
$$

where $c, \mu, a, b$ are the volume, the shear modulus, the thickness and the length of the particle, respectively, $\varepsilon$ is the strain obtained by subtraction of particle plastic relaxation from martensite strain. The value of $\mu$ can be derived from strengthening curves of Fe-based alloys [10]. The estimation $\mu \approx 500 / 0.02=2.5 \times 10^{5} \mathrm{MPa}$ can be obtained, assuming that the strength of the particles is not less than the strength of the aged alloy. One can estimate the strain $\varepsilon$ considering rotation about axis [110] by the angle $5-6^{\circ}$ as a shear strain, i.e. $\varepsilon=2 \times \sin \left(6^{\circ}\right) \approx 0.2$ [10]. Assuming $c=0.1$ the value $E=32 \mathrm{cal} / \mathrm{mol}$ can be calculated for plate particles with the aspect ratio $a / b=0.2$ and $E=160 \mathrm{cal} / \mathrm{mol}$ - for spherical particles.

These values are nearly equal to the energy values needed for MT in Fe-Ni-based alloys. That is why significant decrease of temperature hysteresis during MT is observed. In the framework of the model 
proposed, strain of particles determines the degree of stresses stored independently of strain nature (elastic or structural strain, twinning or plastic shear). The higher volume content of precipitate particles, the more elastic energy is stored in the system. According to the aspect ratio $(a / b)$ in (14), an elastic energy stored in a matrix with spherical particles ( $\gamma^{\prime}$-phase), is higher than the one stored in a matrix with plate particles ( $\gamma^{\prime \prime}$-phase). In addition to this, sizes of particles are essentially important also. Probably, the characteristic scale on which stresses are accommodated elastically, should not be more than value of additional shear with invariant lattice, for example, a width of twins

\section{CONCLUSIONS}

Thus, some characteristics of MT, namely, a wide temperature hysteresis, a temperature range of direct (reverse) martensitic transformation and others can be manipulated using a degree of coherence for parent and martensite phases, a shear stress needed for moving interface boundary, and by estimating elastic stresses in a system with martensite crystals and stress relaxation.

One can arise the question about essential substance of martensitic transformation and some features which distinguish MT from other phase transformations in solid. Apparently, the following features characterize MT as a special class among other phase transformations:

- diffusionless and cooperative shear character of MT;

- equivalency of chemical and elastic constituents of transformation driving force.

That is why martensitic transformation can be defined as a diffusionless shear phase transformation, in which chemical driving force has the same importance as elastic stresses in a system martensite crystals matrix.

\section{Acknowledgments}

This work was partially supported by INTAS grant No. 93-1202 ext.

\section{References}

[1] Kurdjumov G.V., DAN SSSR 60 (1948) 1543-1546; JTF 18 (1948) 999-1025.

[2] Gulyaev A.P., Metallovedenie (Metallurgiya, Moskva, 1978) pp. 109-112.

[3] Likhachev A.A., Koval Yu.N., Scr, Met. et Mat. 27 (1992) 11-15.

[4] Sinclair R., Mohamed H.A., Acta Met. 26 (1978) 623-629.

[5] Koval Yu.N., "Peculiarities of martensitic transformation and parameters of the SME in Fe-based and B2-type-based alloys", Applied Crystallography, Cieszyn, Poland, 22-26 August 1994, H. Morawiec and D. Stroz Eds. (World Scientific Publishing, Singapore, 1995) pp. 223-228.

[6] Roitburd A. L., Solid State Phys.: Adv. Res. and Appl. 33 (1978) 317-390.

[7] Olson G.B., Cohen M., Scr. Met. 9 (1975) 1247-1251.

[8] Koval Yu.N., Kozlov A.P., Monastyrsky G.E., Scr. Met. 23 (1989) 1711-1734.

[9] Paskal Yu.J., Monasevich L.A., Izv. Vuzov. Fizika 11 (1978) 98-103.

[10] Koval Yu.N., Monastyrsky G.E., "On the nature of the variation of martensitic transformation hysteresis and SME characteristics in Fe-Ni-based alloys", ICOMAT'95, Lausanne, Switzerland, 20. 25 August 1995, R. Gotthardt and J. Van Humbeeck Eds. (Les Editions de Physique, France) pp. C\&397-C8-402. 